\title{
Thermo-Elastic Behaviour of Single Ply Triaxial Woven Fabric Composites
}

\author{
A.B.H. Kueh* and S. Pellegrino ${ }^{\dagger}$ \\ University of Cambridge, Cambridge, CB2 1PZ, UK
}

\begin{abstract}
This paper studies the complex thermal deformation of single-ply triaxial weave composites. This behaviour is studied experimentally, by testing flat plates and narrow strips of TWF, and numerically, by carrying out finite-element simulations that capture the effects of the thermo-mechanical anisotropy of the individual tows that make up the composite. It is shown that the dominating effect is the development of a thermally-induced twist.
\end{abstract}

\section{Introduction}

Single-ply Triaxial Woven Fabric (TWF) composites deform in an unexpected way when subjected to uniform thermal loading. This has been known for a while, but not understood. In this paper we study this behaviour experimentally, by testing flat plates and narrow strips of TWF, and numerically, by carrying out finite-element simulations that capture the effects of the thermo-mechanical anisotropy of the individual tows that make up the composite. The two lines of investigation are in broad quantitative agreement, and show that the dominating effect is the development of a thermally-induced twist.

TWF composites have been used for several years for large antenna structures, both rigid and deployable. TWF is made up of yarns woven in three distinct directions, at 0 - and +/-60; it is impregnated with resin and cured like standard composites. Its main attractions are lightness and quasi-isotropic mechanical behaviour. ${ }^{1}$ Hence, it is potentially suitable to form single-ply structural elements of great efficiency. However, difficulties have been encountered in some recent applications of this material, due to unexpected thermally induced deformations occurring in high-precision structures made from single-ply TWF.

This paper focusses on a specific material, described in Section II. To establish some facts about the behaviour of TWF, Section III shows the results of some representative tests on flat, rectangular plates and on narrow strips. Section IV derives the thermo-mechanical parameters for the tows, modelled as transversely isotropic. Section V presents finite-element models of simple woven structures that consist of straight tows, stepped tows, as well as the strips that have been tested. Results from these models, presented in Section VI, show that a surprisingly rich pattern of thermal deformation behaviour is induced by the combination of eccentricity of the tows at the cross-over points, coupled with the anisotropic thermal expansion properties of the tows. The analysis is then extended to structures consisting of non-straight tows, forming a pattern similar to that of TWF.

The reader is referred to Refs. ${ }^{1,2}$ for a more detailed introduction to TWF composites.

\section{Material Description}

The material investigated in this paper is based on the SK- 802 fabric, manufactured by Sakase-Adtech Ltd., Japan. This particular fabric comprises 1000 filaments of T300 carbon fibre, woven in the 'basic weave' pattern shown in Figure 1. About half of the area is covered by hexagonal holes. This weave has a dry areal density of $75 \mathrm{~g} / \mathrm{m}^{2}$ and a thickness of about $0.15 \mathrm{~mm}$.

The material is processed as follows. TWF is laid up underneath one layer of $40 \mathrm{~g} / \mathrm{m}^{2}$ semi-solid resin film, on a release paper, for a single side infusion. The resin used is Hexcel 8552 (a space-qualified resin). The lay-up is cured in an autoclave at a temperature of $185^{\circ} \mathrm{C}$ and a pressure of 6 bar, for 2 hours.

\footnotetext{
*Research student, Department of Engineering, Trumpington Street.

${ }^{\dagger}$ Professor of Structural Engineering, Department of Engineering, Trumpington Street. Associate Fellow AIAA.
} 
Table 1. Fiber and matrix material properties

\begin{tabular}{|l|c|c|}
\hline & T300 & Hexcel 8552 \\
\hline Density, $\rho\left(\mathrm{kg} / \mathrm{m}^{3}\right)$ & 1,760 & 1,301 \\
\hline Longitudinal stiffness, $E_{1}\left(\mathrm{~N} / \mathrm{mm}^{2}\right)$ & 230,000 & 4,570 \\
\hline Transverse stiffness, $E_{2}\left(\mathrm{~N} / \mathrm{mm}^{2}\right)$ & 14,000 & 4,570 \\
\hline Shear stiffness, $G_{12}\left(\mathrm{~N} / \mathrm{mm}^{2}\right)$ & 8,960 & 1,668 \\
\hline Poisson's ratio, $\nu_{12}$ & 0.2 & 0.37 \\
\hline Coefficient of thermal expansion $(\mathrm{CTE}) \alpha\left(/{ }^{\circ} \mathrm{C}\right)$ & $-0.41 \times 10^{-6}$ & $65 \times 10^{-6}$ \\
\hline
\end{tabular}

The properties of the fibres and the matrix are listed in Table 1.

A micrograph showing the tow cross-sections can be seen in Figure 2. The exact shape and orientation vary depending on where the section is taken, but in general he cross-sections consist of a central part, roughly of constant height, tapering to a point on either side. The average tow width, measured from a series of micrographs, is $0.85 \mathrm{~mm}$.

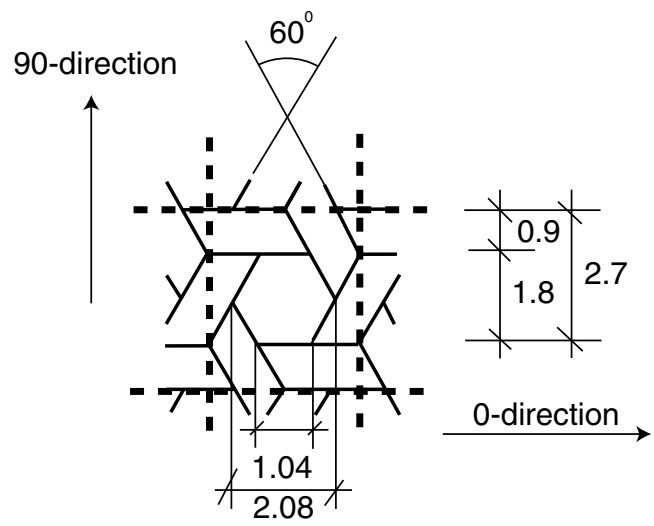

Figure 1. Triaxial woven fabric unit cell (dimensions in $\mathbf{m m})$.

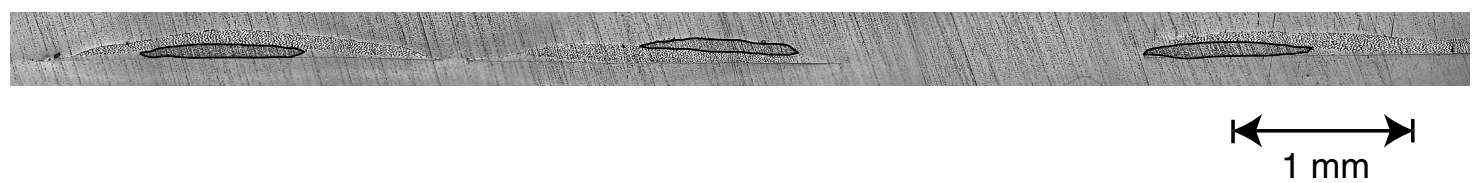

Figure 2. Micrograph of tow cross section

\section{Thermal Tests}

\section{A. Apparatus}

Previous thermal tests ${ }^{1}$ were carried out in a furnace with a glass door. When the same apparatus was used to carry out more careful experiments, in which the time response of the specimens was monitored, significant time-dependent behaviour was observed. The slow time response of the furnace was deemed to be a significant contributor. In order to minimize this effect, and so make progress in understanding the time-independent response of the material before moving on to other effects, a different apparatus that uses radiation to quickly heat the specimen was devised. The basic idea is to use a floodlight, controlled through a variable resistor to illuminate a black, flat steel plate and to hold the specimen close (about $5 \mathrm{~mm}$ ) to this 
hot plate. The plate quickly heats up thus in turn heating the specimen (it also acts as a cover to prevent the light from the floodlight going into camera lens). An infrared spot temperature meter is used to measure the temperature on the specimen surface, and the variable resistor is adjusted accordingly. The temperature of the samples has been found to be quite uniform.

The experiment setup is shown in Figure 3. The deformation of the specimen, is measured from a series of digital photographs, using photogrammetry software. For this purpose, a number of targets are attached to the specimen surface. Pictures are taken at various angles at certain temperatures to capture the three-dimensional distortion of the specimen.

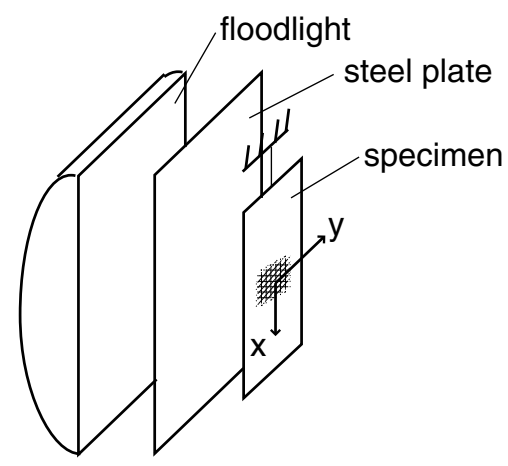

Figure 3. Thermal test set up

\section{B. Results}

A first set of experiments was performed on $90 \mathrm{~mm}$ wide $\times 190 \mathrm{~mm}$ long samples. The samples were hung from a single point, in front of the heated plate, with the 0-direction of the material (see Figure 1) vertical, i.e. in the $x$-direction see Figure 3 . Samples cut at 90 degrees were also tested; the results were equivalent and will not be discussed.

The samples were heated to 60,80 , and $100^{\circ} \mathrm{C}$ and then allowed to cool naturally. The typical heating time from $20^{\circ}$ to $100^{\circ}$ was $40 \mathrm{~s}$ and the cooling back to $20^{\circ}$ took 15 minutes. Regular measurements of the shapes of the plates were taken, both during heating and cooling, and the curvatures $\kappa_{x}$ and $\kappa_{y}$ of the plate were obtained by fitting polynomial deflected shapes through the measured deflections. Figure 4 shows representative plots of the curvatures $\kappa_{x}$ and $\kappa_{y}$ at the centre point of a plate, against temperature.

In each test the plate is approximately straight in the $x$-direction and slightly curved in the $y$-direction. As the temperature is increased, $\kappa_{x}$ remains approximately constant whereas the magnitude of $\kappa_{y}$ increases approximately linearly until the temperature reaches a maximum. Then, while the plate is cooling, the magnitude of $\kappa_{y}$ continue to increase and and reaches a maximum approximately proportional to the temperature increment, finally, $\kappa_{y}$ decreases over a period of several hours while the temperature is constant.

This experiment shows clear signs of visco-elastic material behaviour during cooling, i.e. during the slow part of the cycle. However, the behaviour observed is quite complex and, rather than attempting to explain it, we will present a second sets of experimental results which will provide a more direct clue to the behaviour just described.

To remove some of the complexity shown by the first set of tests, the second set was performed on narrow strips of TWF cut either in the 0-direction or in the 90-direction. Here we present results obtained from $59 \mathrm{~mm}$ long specimens. Because the unit cell is not square the 0-direction specimen contains 20 hexagons whereas the 90-direction specimen contains 12 units.

The specimens were clamped to a rigid support at one end and were held parallel and close to the radiating plate as for the previous test. The specimens were painted white on one edge, and the deflection of this edge was measured from a series of photographs.

Figure 5 shows two views of the same edge, at $25^{\circ}$ and at $120^{\circ}$. We were surprised to discover that the edge remains straight in the deformed configuration and, since this observation was confirmed on several specimens both in the 0-direction and in the 90-direction, we conjectured that the thermal deformation of TWF strip is a uniform twist. 


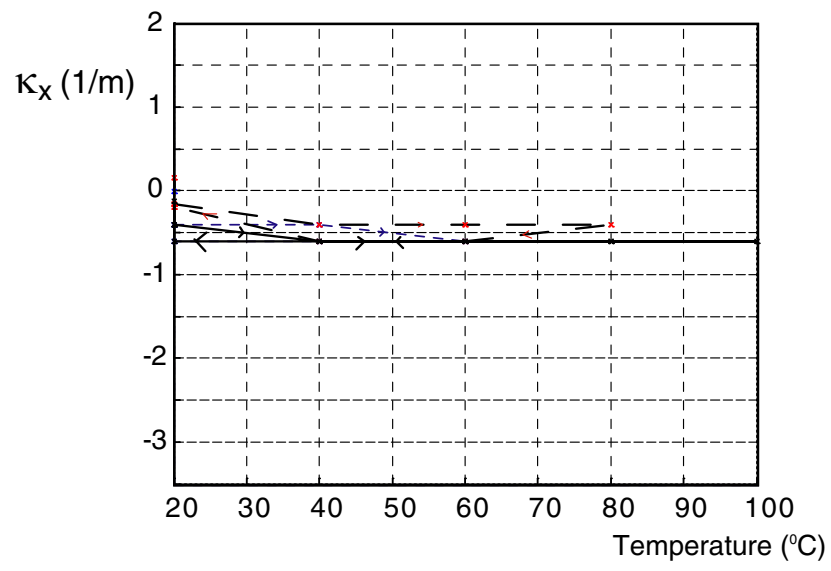

(a)

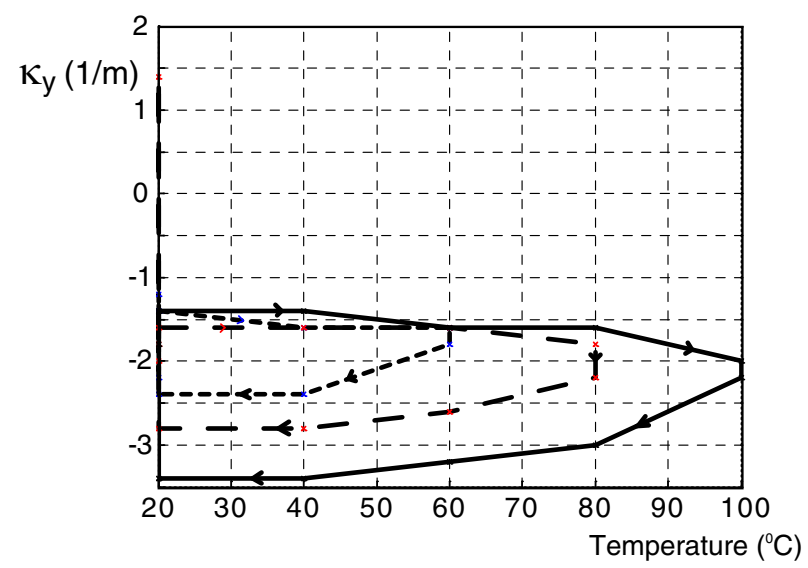

(b)

Figure 4. Curvature (a) $\kappa_{x}$ (b) $\kappa_{y}$ vs temperature in a rectangular plate with 0-direction vertical.

(a)
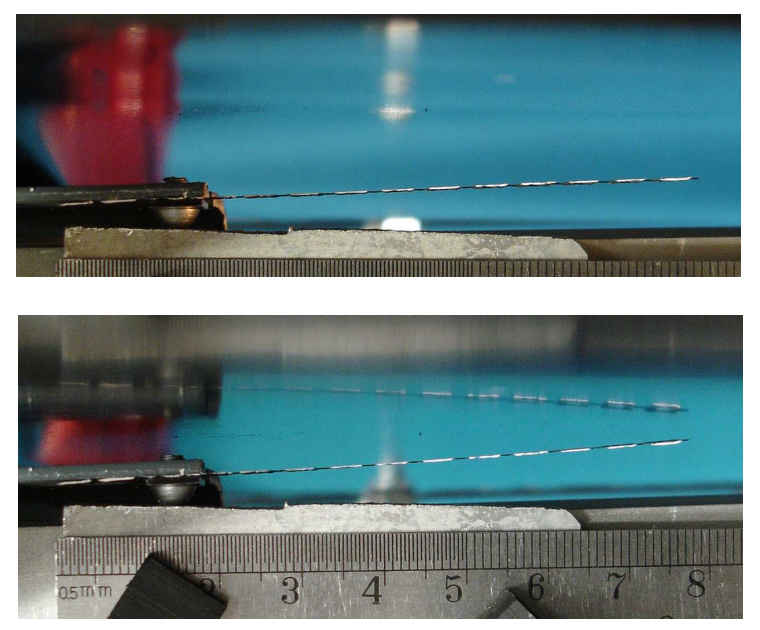

Figure 5. Edge deformation of TWF cantilever strip (a) at $25^{\circ}$ and (b) at $120^{\circ}$. 
Figure 6 contains plots of the end displacements of two different strips, at different temperatures.

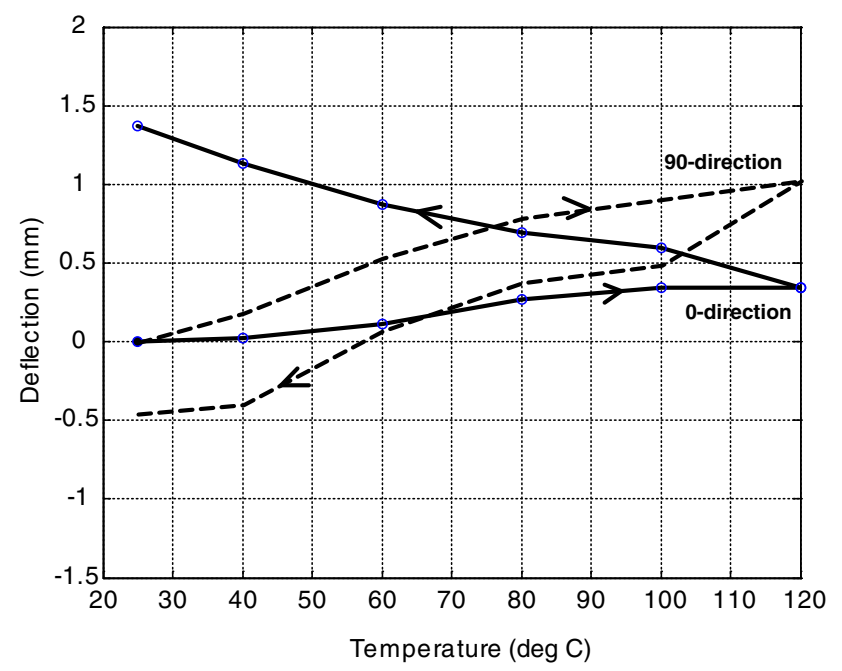

Figure 6. Tip deflections of TWF cantilever strips.

\section{Tow Properties}

Each tow is modelled as a three-dimensional continuum having transversely isotropic properties; the modulus in the fibre direction is higher than the two orthogonal directions, and the transverse moduli are equal. The number of independent elastic constants needed to model this continuum is five: the longitudinal stiffness, $E_{1}$, the transverse stiffness, $E_{2}$, the longitudinal Poisson's ratio, $\nu_{12}$, and the shear moduli, $G_{12}$ and $G_{23}$. The thermal properties of the tow are also assumed to be transversely isotropic.

The independent engineering constants are determined as follows. ${ }^{3}$ The longitudinal extensional modulus $\mathrm{E}_{1}$ is obtained from the rule of mixtures

$$
E_{1}=E_{1 f} V_{f}+E_{m}\left(1-V_{f}\right)
$$

where the subscripts $f$ and $m$ denote fibre and matrix, respectively. The Poisson's ratio is also found from the rule of mixtures

$$
\nu_{12}=\nu_{13}=\nu_{12 f} V_{f}+\nu_{m}\left(1-V_{f}\right)
$$

The transverse extensional modulus is found from the Halpin-Tsai semi-empirical relation

$$
E_{2}=E_{3}=E_{m} \frac{1+\xi \eta V_{f}}{1-\eta V_{f}}
$$

where

$$
\eta=\frac{E_{2 f}-E_{m}}{E_{2 f}+\xi E_{m}}
$$

and the parameter $\xi$ has been set equal to 2.0 has been chosen. ${ }^{3}$ The shear modulus $G_{12}=G_{13}$ is found from the Halpin-Tsai semi-empirical relation ${ }^{3}$

$$
G_{12}=G_{13}=G_{m} \frac{\left(G_{12 f}+G_{m}\right)+V_{f}\left(G_{12 f}-G_{m}\right)}{\left(G_{12 f}+G_{m}\right)-V_{f}\left(G_{12 f}-G_{m}\right)}
$$

The derivation of the in-plane shear modulus $G_{23}$ is based on Ref. ${ }^{4}$ The details are rather lengthy and are not shown here. Finally, $\nu_{23}$ is computed from

$$
G_{23}=\frac{E_{2}}{2\left(1+\nu_{23}\right)}
$$

$$
5 \text { of } 12
$$


The longitudinal thermal expansion coefficient is derived from ${ }^{5}$

$$
\alpha_{1}=\frac{E_{1 f} \alpha_{f} V_{f}+E_{m} \alpha_{m} V_{m}}{E_{1 f} V_{f}+E_{m} V_{m}}
$$

and the transverse thermal expansion coefficient from ${ }^{5}$

$$
\alpha_{2}=\alpha_{3}=V_{f} \alpha_{f}\left(1+\nu_{12 f}\right)+V_{m} \alpha_{m}\left(1+\nu_{m}\right)-\left(V_{f} \nu_{12 f}+V_{m} \nu_{m}\right) \alpha_{1}
$$

Using the measured volume fraction, $V_{f}=0.61$, the engineering constants for a tow made of T300/Hexcel8552 have been computed and are listed in Table 2 .

Table 2. T300/Hexcel 8552 tow material properties

\begin{tabular}{|l|c|}
\hline Longitudinal stiffness, $E_{1}\left(\mathrm{~N} / \mathrm{mm}^{2}\right)$ & 142,082 \\
\hline Transverse stiffness, $E_{2}=E_{3}\left(\mathrm{~N} / \mathrm{mm}^{2}\right)$ & 9,106 \\
\hline Shear stiffness, $G_{12}=G_{13}\left(\mathrm{~N} / \mathrm{mm}^{2}\right)$ & 4,069 \\
\hline In-plane shear stiffness, $G_{23}\left(\mathrm{~N} / \mathrm{mm}^{2}\right)$ & 4,043 \\
\hline Poisson's ration, $\nu_{12}=\nu_{13}$ & 0.2663 \\
\hline Longitudinal CTE, $\alpha_{1}\left(/{ }^{\circ} \mathrm{C}\right)$ & $0.41 \times 10^{-6}$ \\
\hline Transverse $\mathrm{CTE}, \alpha_{2}\left(/{ }^{\circ} \mathrm{C}\right)$ & $34.30 \times 10^{-6}$ \\
\hline
\end{tabular}

\section{Finite Element Models}

The key element of our simulation, carried out with the ABAQUS finite-element package, is the model of a single tow as a 3D continuum with the properties defined in Table 2. The tow has uniform, rectangular cross-section, $0.078 \mathrm{~mm}$ thick and $0.803 \mathrm{~mm}$ wide. The thickness was obtained by halving the measured thickness of TWF samples, and the width by setting the area of the rectangular model equal to the average tow cross-sectional area, determined from several micorgraphs.

An 8-node linear brick element with incompatible modes, C3D8I, is used to model the tows. The incompatible modes included in the formulation of this element lead to better performance in bending, and hence a lower mesh density is needed to achieve convergence compared to a standard 8-node linear brick element. Small triangular regions in the 90-direction model are meshed with the 6-node linear triangular prism, C3D6. They are also less time-consuming compared to higher order brick elements. The mesh density of aforementioned models are listed in Table 3. In the first model (cross plate) there are only two elements in the through-thickness direction. All other models have four elements through the thickness.

Table 3. Model mesh density

\begin{tabular}{|l|c|c|}
\hline Model configuration & Node number & Element Number \\
\hline Cross plate & 10,325 & 6,912 \\
\hline Rectangle & 680 & 384 \\
\hline Hexagon (flat plate) & 4,050 & 2,304 \\
\hline X-configuration & 1,575 & 1,024 \\
\hline Woven unit cell & 1,800 & 1,152 \\
\hline 0-strip & 34,975 & 25,728 \\
\hline 90-strip & 27,295 & 19,200 \\
\hline
\end{tabular}

First, we consider a simple model consisting of two straight tows at right angles that are fully bonded across the contact region, see Figure $7(\mathrm{a})$.

Next we consider two pairs of straight, parallel tows forming a two-up two-down square, Figure 7(b). Then, we consider three pairs of parallel tows forming a one-up, one-down, one-up, etc. hexagon, Figure $7(\mathrm{c})$. A gap of $0.169 \mathrm{~mm}$ separates the tips of the tows. 
(a)
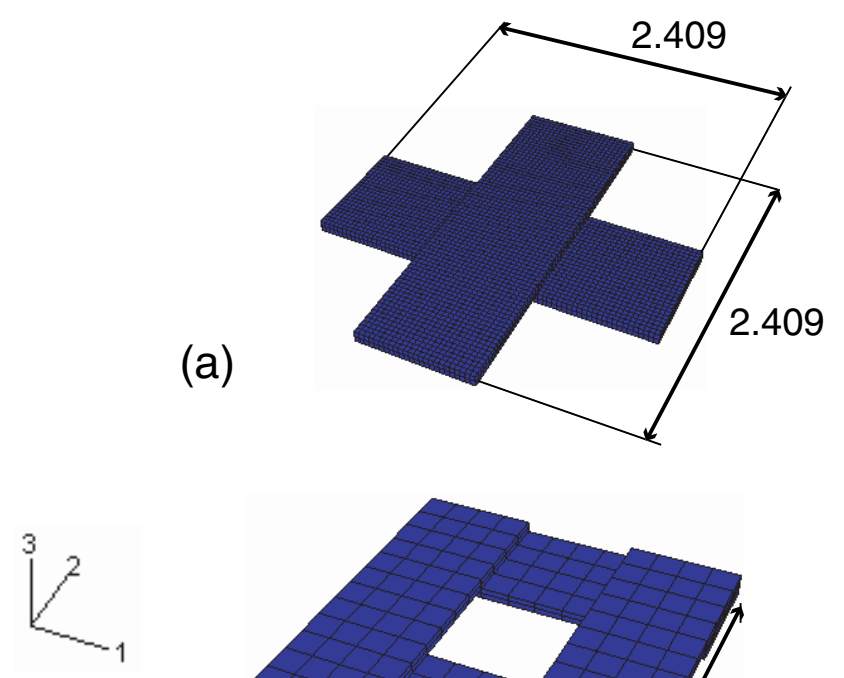

(b)
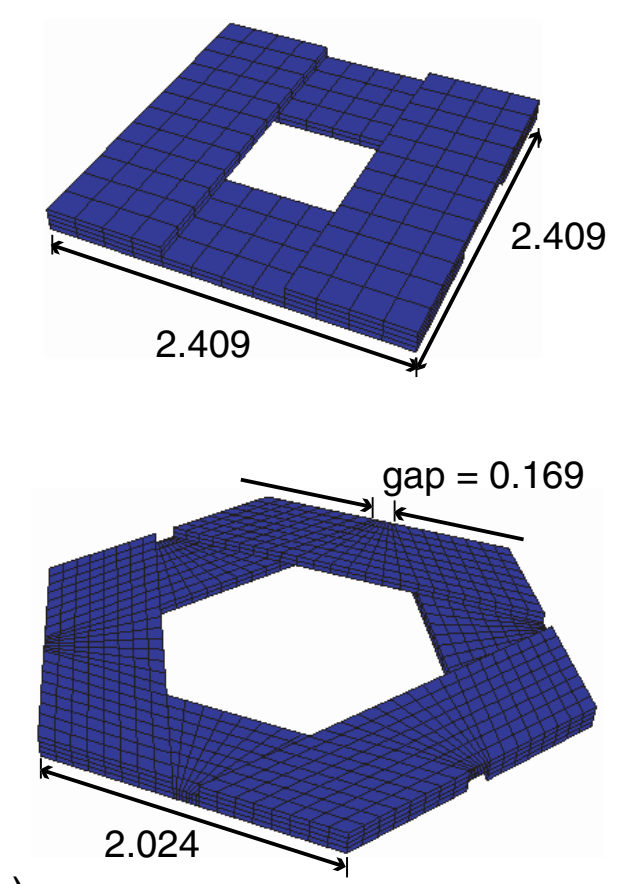

(c)

Figure 7. (a) Tows at right angles (b) square and (c) hexagon (dimensions in mm). 
Finally, to model the weave geometry, a stepped tow is used. Two configurations, forming an $\mathrm{X}$ and a kind of woven hexagon, see Figure 8, are considered.

The key difference between the hexagon consisting of stepped tows, in Figure 8(c) and the earlier hexagon in Figure $7(\mathrm{c})$ is that this last configuration has an axis six-fold symmetry but has no mirror symmetry. Note that the tows gradually climb up and suddenly step down if one goes round the hexagon in an anticlockwise sense. The gap dimension in this model is the same as before.

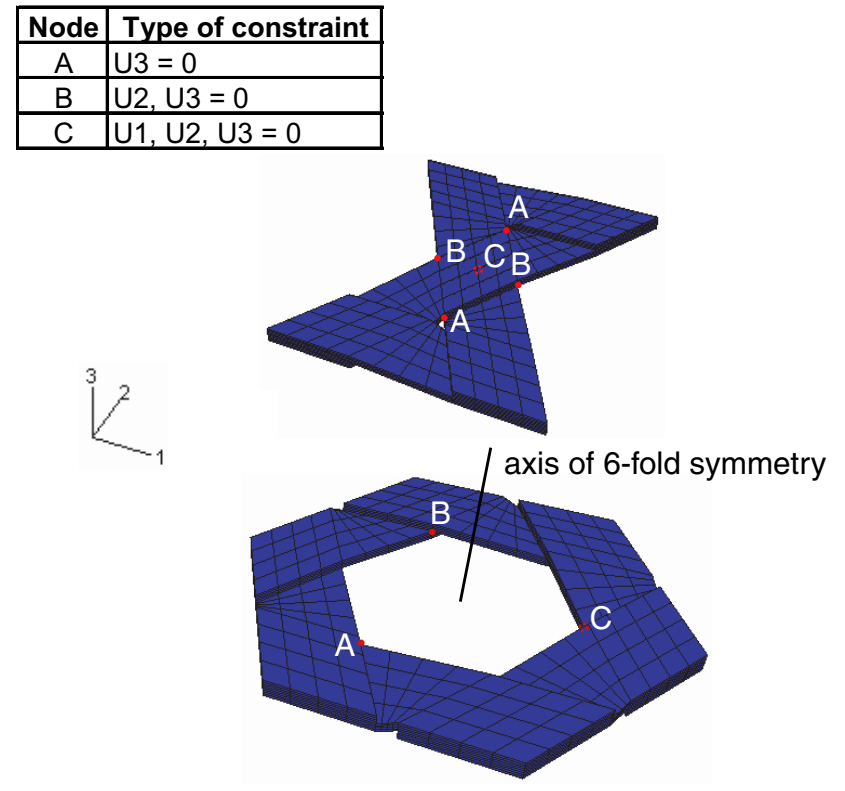

Figure 8. (i) X-plates (ii) stepped-tow hexagon.

The last model that is considered is a strip consisting of multiple unit cells, arranged either in the 0direction or the 90-direction. There are 20 hexagon units and 10 unit cells in these strips, respectively.

A uniform temperature increase of $100^{\circ} \mathrm{C}$ is imposed on all models. The mechanical boundary conditions are free-free for the straight-tow models, whereas the boundary conditions for the stepped-tow models are shown in Figure 8. The same boundary conditions of Figure 8(ii) are also applied to the strip models. The cross over regions are connected by tieing together all nodes on the contact surfaces against both translation and rotation. A linear elastic analysis is performed in all cases.

\section{Results}

It is interesting to begin by considering the deformation of the contact surface between the two tows in the simple cross model. Our analysis shows that this initially flat surface deforms into a saddle shape, as can be seen from the plot of Gaussian curvature of this surface, Figure 10, which is everywhere negative.

Figure 11 shows contour plots of the out-of-plane displacement of the top surface of the square and hexagonal structure formed from straight tows. It can be seen that the inner part and also the corners do not move, but the edges deform alternately up and down.

Similar plots are shown in Figure 12 for the stepped tow X-structure and hexagon. However this time the deformation mode of the edge of the structure involves the same edge going up and then down. Hence the wavenumber has doubled.

Figure 13 shows the edge deflection of 0-direction and 90-direction strips. In the case of the 0-direction strip there is a continuous tow along the edge, and it turns out that this tow deforms into a wave, whose characteristic length matches the stepped-tow hexagon result. In the case of the 90-direction tow there is no continuous structure along the edge and, depending on which set of points we choose to plot, the outcome is somewhat different in detail.

However, the overall effect is that the edges of the strips of TWF remain essentially straight when the 


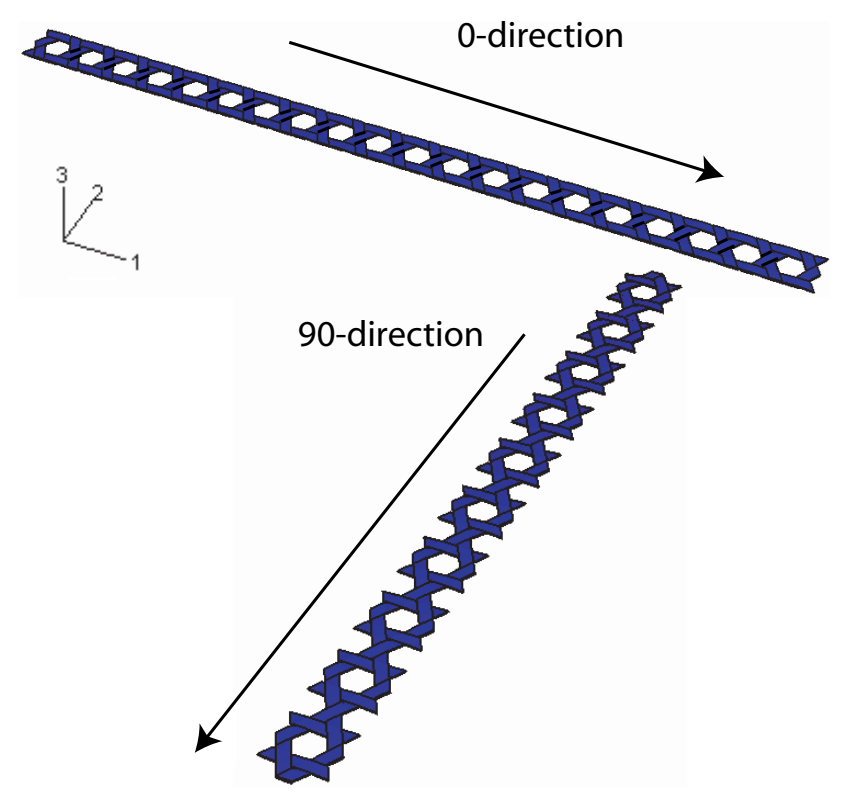

Figure 9. Models of TWF strips in 0-directions and 90-direction.

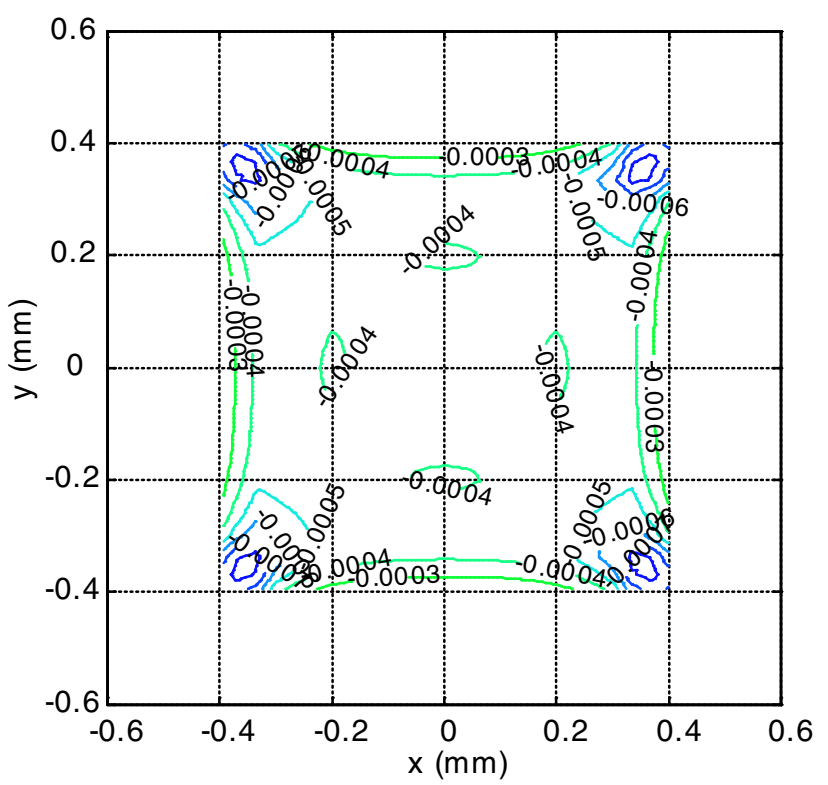

Figure 10. Gaussian curvature of interface between two tows. 

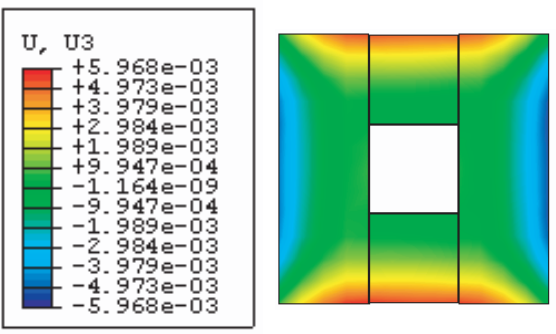

(a)
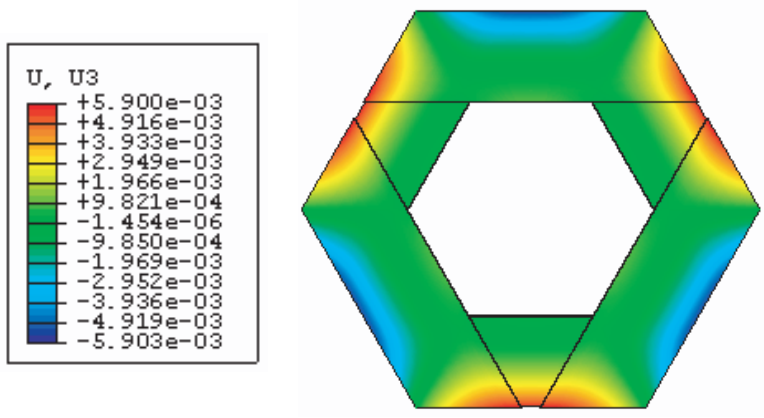

(b)

Figure 11. Out of plane displacement contour plot of (a) square and (b) hexagonal structure.

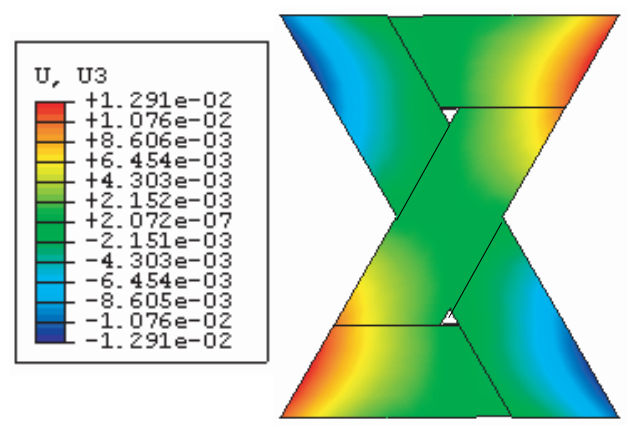

(a)

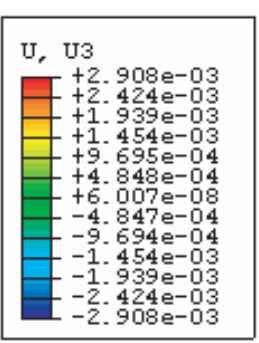

(b)

Figure 12. Out of plane displacement contour plot of (a) X-configuration and (b) woven unit cell 


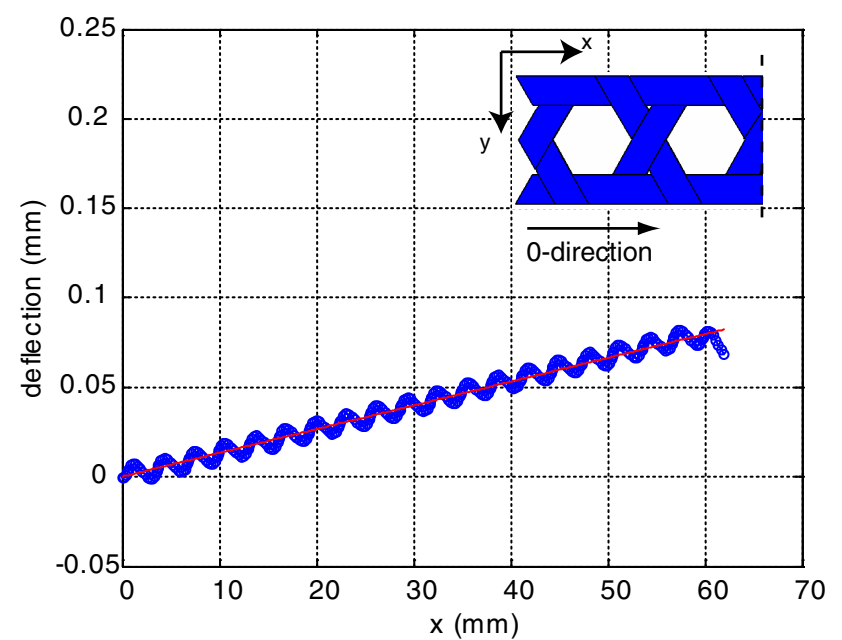

(a)

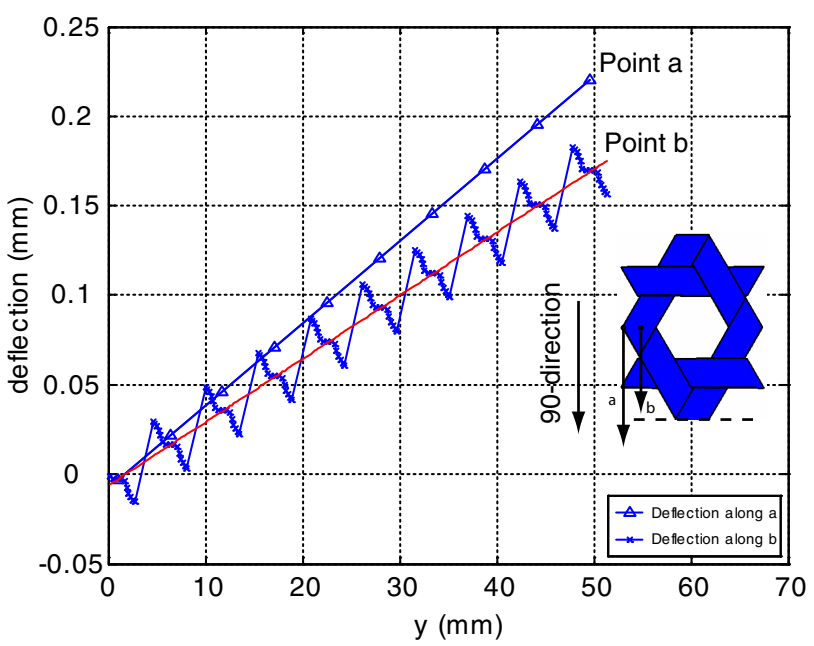

(b)

Figure 13. Edge deflection of (a) 0-direction strip and (b) 90-direction strip subject to a 100 degree temperature increase. 
strips are heated, which agrees with the experimental observations in Section III.

\section{Discussion}

Our simulation appears to have captured a key element from the experimental study, namely the straightness of the thermally deformed strips of TWF. However, our predictions are rather smaller than the measured deflections.

Comparing the tip deflections in Figure 13 with the linear part of the measured response in Figure 6 , and allowing for the difference in the temperature ranges, one finds that our estimates are too small by a factor of about 5. However, if the comparison is made between the ratio between the tip deflection of the 0-direction strip against that of the 90-direction strip, one finds that the ratios are within $20 \%$.

There are several possible explanations for these discrepancies. First, the tow parameters used in our simulation were not obtained from direct measurements and, for example, changing the fibre volume fraction significantly affects the deflection, see Figure 14

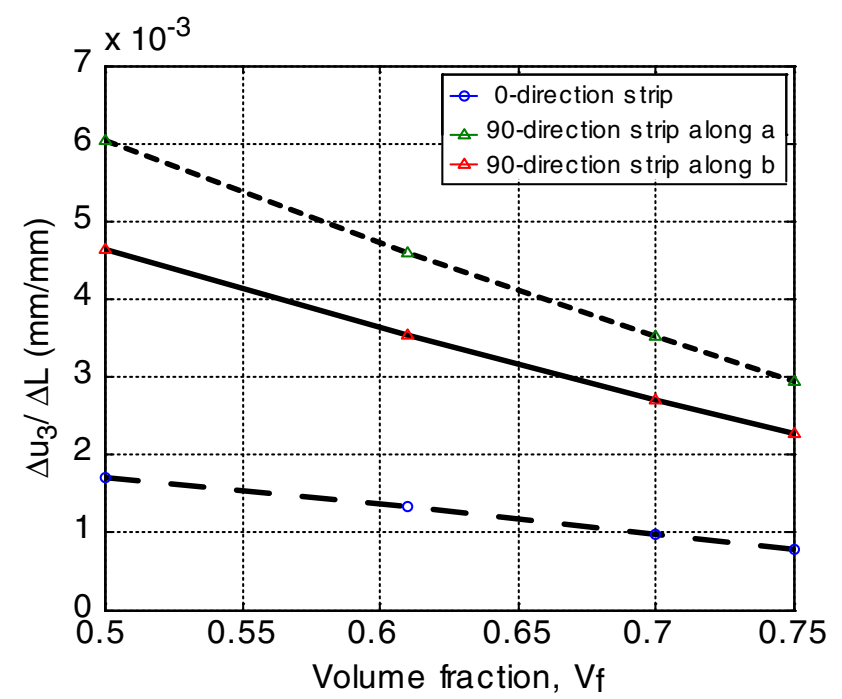

Figure 14. Change of deflection per unit length with volume fraction.

Also, the tow cross-sections have been modelled as rectangular which leads to the cross-over regions being initially flat. The effects of this assumption need to be analysed.

Finally, visco-elastic behaviour of the matrix, which is clearly visible in our experimental results needs to be included in future models. Alternatively, the use of a more stable matrix should be considered.

\section{Acknowledgements}

AK thanks Universiti Teknologi Malaysia for financial support. We are grateful to Mr R. Sakai of Sakase Adtech for providing the material SK-802 and extensive information on it, Mr J. Ellis of Hexcel, Duxford for providing materials and support in the preparation of specimens.

\section{References}

\footnotetext{
${ }^{1}$ Kueh, A., Soykasap, O., and Pellegrino, S. Thermo-mechanical behaviour of single-ply triaxial weave carbon fibre reinforced plastic. European Conference on Spacecraft Structures, Materials and Testing, 9-13 May 2005, ESA Estec.

${ }^{2}$ Zhao, Q., and Hoa, S. V. (2003). Thermal deformation behavior of triaxial woven fabric (TWF) composites with open holes. Journal of Composite Materials, 37(18), 1629-1649.

${ }^{3}$ Daniel I.M., Ishai O., 1994, Engineering Mechanics of Composite Materials, Oxford University Press.

${ }^{4}$ Whitney J.M., Daniel I.M., Pipes R.B., 1984, Experimental Mechanics of Reinforced Composite Materials, Society for Experimental Mechanics.

${ }^{5}$ Tsai, S.W. and Hahn, H.T., Introduction to conmposite materials, Technomic, 1980.
} 